\title{
Modeling and Simulation of a Moving Yarn Segment: Based on the Absolute Nodal Coordinate Formulation
}

\author{
Shujia Li, ${ }^{1}$ Yongxing Wang $\mathbb{D}^{1,2}{ }^{1,2}$ unxun $\mathrm{Ma}^{1}{ }^{\text {and Shengze Wang }}{ }^{1,2}$ \\ ${ }^{1}$ College of Mechanical Engineering, Donghua University, Shanghai 201620, China \\ ${ }^{2}$ Research Center of Advanced Textile Machinery, Ministry of Education, Donghua University, Shanghai 201620, China
}

Correspondence should be addressed to Yongxing Wang; wangyx@dhu.edu.cn

Received 13 March 2019; Accepted 9 July 2019; Published 25 July 2019

Academic Editor: Gaetano Giunta

Copyright (c) 2019 Shujia Li et al. This is an open access article distributed under the Creative Commons Attribution License, which permits unrestricted use, distribution, and reproduction in any medium, provided the original work is properly cited.

\begin{abstract}
A new finite element dynamic model of a moving yarn segment has been proposed in this paper based on the absolute nodal coordinate formulation (ANCF). Apart from taking into account the elastic properties of the yarn in three dimensions, the model also considers the viscosity in the longitudinal direction and takes into account the effect of gravity and air resistance. In this paper, the simulation described the movement of the yarn segment that is pulled by the fixer on the guideway. Then, a corresponding experiment was proposed to evaluate the theoretical model. The theoretical and experimental comparisons of the motion tracing exhibited good agreement, demonstrating that the new model could predict the actual moving trace of the yarn segment. Moreover, another simulation of the spatial motion of the yarn segment was presented, to elucidate the role of the model in predicting the movement of the yarn segment. After considering the parameters of the actual process and its constraints, the authors established that the proposed model could be used to predict the trajectory of a yarn segment in the actual production process, which is vital when fabricating textile products.
\end{abstract}

\section{Introduction}

As the intermediate component and fundamental constituent of the process of turning fiber into fabric in the textile industry, the quality of the yarn directly affects the quality of the final textile product. There are different types of yarn, such as twisted yarns or continuous filaments that are not twisted. Hereafter, in this paper, both types have been collectively referred to as "yarn".

In the process of producing textiles, in addition to the continuous moving yarn, there is another state in which the yarn is clamped by a machine at one end and the rest of the yarn keeps moving under the pulling action of the machine, for example, a yarn segment that protrudes from the nozzle of the machine during air-jet weft insertion (Figure 1(a)) or during the pneumatic yarn splicing process, or a yarn segment on the bobbin when the yarn is broken during spinning (Figure 1(b)), or a yarn segment at the start of the filament winding process (Figure 1(c)). The feature that is common to all of these yarn segments is that one end of the yarn is pulled by a mechanical device to complete various types of movement. Therefore, the actual movement of the yarn segment which is held by the gripper is critical, and the success of this action is determined by whether the yarn can move according to the planned path.

Of the above cases, yarn splicing, especially during the pneumatic yarn splicing process and the coupling of the yarn and the airflow, is of particular concern to researchers. However, when studying the yarn-breaking and filament winding starting processes, researchers focused on the dynamics of the mechanical parts rather than looking at the process from the perspective of the yarn. In addition to the pneumatic yarn splicing process, automatic yarn-splicing robots are now a research hotspot with regard to the spinning process. For this process, the yarn segments are not only coupled with the airflow, but this also includes different types of motions as the yarn is pulled by the gripper. Currently, few researchers have investigated this topic.

The modeling and simulation of the yarn begin with the ball-chain model in the early stage of research [1-5]. Then the authors presented that modeling fibers with fewer elongated bodies, as opposed to many spheres, significantly reduced 


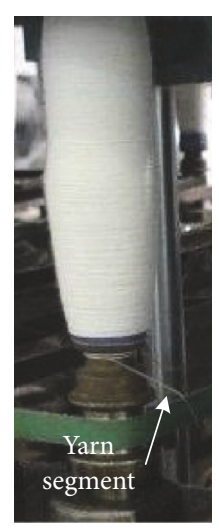

(a)

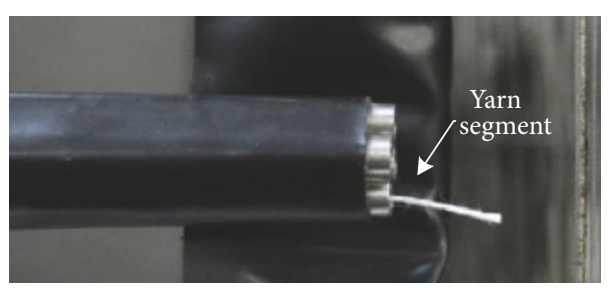

(b)

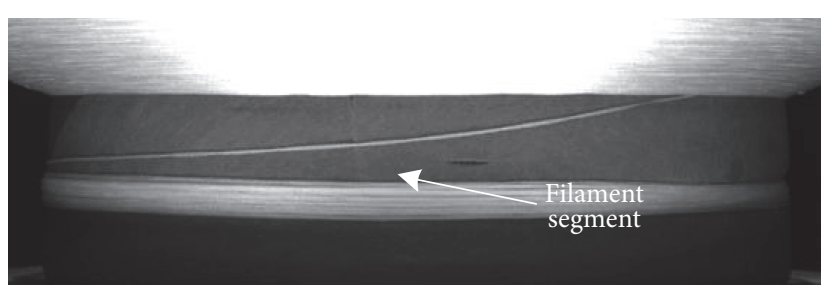

(c)

Figure 1: Different yarn segments in the textile process: (a) yarn segment in the spinning when yarn-breaking; (b) yarn segment in the spinning when yarn-breaking; (c) yarn segment in filament winding starting process.

computation time and facilitated the study of interacting long fibers [5]. This prompted researchers to use the slender body unit to simulate the modeling of yarns. Swop and Ames used the moving string model to study the movement of the yarn during the textile production process [6]. Stanislav Pracek studied the yarn unwinding process based on the string motion theory [7]. The dynamic string model is widely used for analyzing the characteristics of the yarn when it is moving in the longitudinal direction such as vibration. In addition to moving strings, the rope, rod, and beam elements are also typical slender bodies. A study [8] used a rod element and frictionless pins to create a digital chain, which was used to describe the yarn, but it had massive computation. The application of beam element in textile modeling research is mostly used to establish yarn element in fabrics [9]. Gao et al. [10] proposed that because fiber tows length was much higher than their transverse dimensions, beam elements seemed to be the most convenient structural finite element tool; the authors introduced a new 3D beam element with section changes for large-scale textile modeling, but it still stayed on the small transformation case.

The movements of the yarn segments are threedimensional, and, during the movement, the yarn segment undergoes deformation such as stretching, torsion, bending, and shearing in the various processes, and they are affected by air resistance. A three-dimensional description is needed to fully consider the yarn's deformation and motion in the longitudinal direction as well as its cross-section dimensions. This has led to an exciting attempt to simulate the yarn segment using a three-dimensional beam element. Meanwhile, the viscosity of the yarn in the longitudinal dimension, which is the main direction of tension, cannot be ignored in the analysis. In finite element beam theories, there are classical models that come from Euler-Bernoulli's and Timoshenko's theories. As the beam becomes less and less slender or flexible, its mechanics is increasingly threedimensional, and the classical models cannot yield accurate results [11]. Thus, over the years, much effort has been put to enhance and refine the classical beam theories [12], for example, refined beam theories $[11,13]$, beam analysis via hierarchical finite element approach [14], or fiber beam elements [15]. The description methods of the beam theories mentioned above are suitable for structural vibration analysis and composite beam, and there is a more suitable description method for the large-motion, very flexible objects such as yarn, that is, absolute nodal coordinate formulation (ANCF) description. The ANCF beam element has that automatically captured cross-sectional and shear deformations. On the other hand, and in contrast to other large deformation formulations, the equations of motion contain constant mass matrices and generalized gravity forces as well as zero centrifugal and Coriolis inertial forces [16-23]. In fact, the 3D ANCF beam element has been used for the dynamic simulation of spacecraft with simple circular cross-sectional structures [24].

Based on the ANCF, the moving yarn segment element was then established, that is, the yarn segment which is pulled and moves. The simulation of the movement and deformation of the moving yarn segment, with the end node point pulling motion, was then carried out. The effects of gravity on the yarn and air resistance were taken into consideration during this process. When modeling the moving element of the yarn segment, the elastic characteristics of each direction were fully considered, and the viscous characteristics in the longitudinal direction were also computed, so that the established model was more consistent with the actual conditions. Finally, in order to show the validity of the model, a corresponding experiment was conducted. This new finite element dynamic model can be used to analyze the movement and deformation of a yarn segment in textile processes, such as air-jet weft insertion, the yarn piecing up process, and the filament winding starting process. The ultimate goal of this research was to ensure that the yarn moves as expected.

\section{Mathematical Model}

\subsection{Dynamic Model}

2.1.1. Description of the Moving Yarn Segment. Yarn is thin but has a considerable length to diameter ratio. In order 


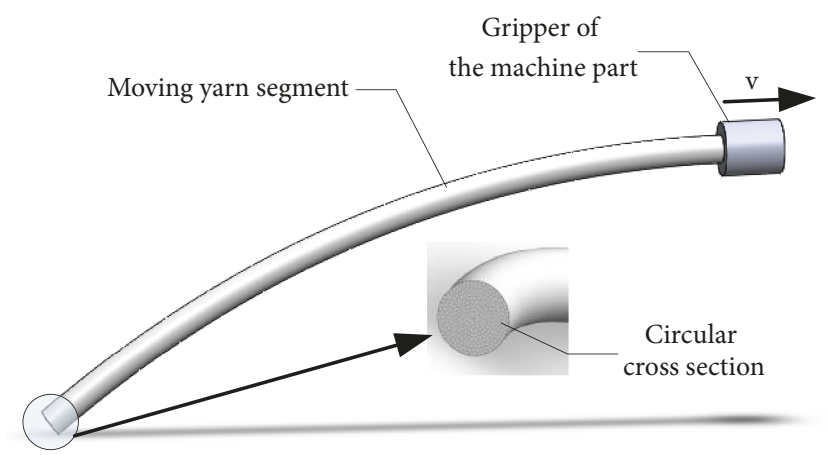

FIGURE 2: The moving yarn segment.

to create a generally described model, it was assumed that the yarn was an ideal continuum with a circular crosssection, as shown in Figure 2. Based on this assumption, all kinds of yarns are can be described with their diameter and length parameters. It should be noted that the cross-sectional diameter and the length of the yarn are the equivalent parameters used for the mathematical model of the moving yarn segment. These equivalent parameters can be obtained by experimental methods or calculated using the following physical parameters: the linear density of the yarn, the twist per unit length, and the parameters of the fiber material. Figure 2 shows the simplified model of the moving yarn segment in the textile industry process.

In this section, the theoretical model of the moving yarn segment has been established based on the ANCF [16], starting with the element model of the moving yarn segment.

2.1.2. Element Model. In this section, the three-dimensional element of a moving yarn segment has been proposed based on the ANCF [16]. The element has been described in the global co-ordinate frame OXYZ. Each element has two nodes, as shown in Figure 3.

As shown in Figure 3, the element of the moving yarn segment has an ideal circular cross-section. The axial length of the element is $l_{e}$, and points $A$ and $B$ represent the nodes of the element. The local element spatial coordinates oxyz were established using node $A$ as the origin, $x$ is the tangent along the longitudinal axis of the element, and $y$ and $z$ were defined in the transverse directions. The displacement and slope were employed as the nodal coordinates of an element, so the nonvector rotational parameters could be avoided. There were 12 coordinate components of each node and 24 components of the element using the ANCF which can be written as

$$
\mathbf{e}=\left\{\begin{array}{llllllll}
\mathbf{r}_{A}^{\mathrm{T}} & \mathbf{r}_{A, x}^{\mathrm{T}} & \mathbf{r}_{A, y}^{\mathrm{T}} & \mathbf{r}_{A, z}^{\mathrm{T}} & \mathbf{r}_{B}^{\mathrm{T}} & \mathbf{r}_{B, x}^{\mathrm{T}} & \mathbf{r}_{B, y}^{\mathrm{T}} & \mathbf{r}_{B, z}^{\mathrm{T}}
\end{array}\right\}^{\mathrm{T}}
$$

where $\mathbf{r}_{A}$ and $\mathbf{r}_{B}$ are the global position vectors of nodes $A$ and $B$, there are three global position components in each node respectively, and $\mathbf{r}_{A, a}$ and $\mathbf{r}_{B, a}$ are the position vector gradients, $a=x, y, z$ defined as $\mathbf{r}_{A, a}=\partial \mathbf{r}_{A} / \partial a$ and $\mathbf{r}_{B, a}=$ $\partial \mathbf{r}_{B} / \partial a$. The global position vector $\mathbf{r}$ of the arbitrary point
$P$ at the moment $t$ can be obtained from the coordinate e:

$$
\mathbf{r}(x, y, z, t)=\mathbf{S}(x, y, z) \mathbf{e}(t)
$$

where $\mathbf{S}$ is the global element shape function matrix.

$$
\mathbf{S}=\left[\begin{array}{llllllll}
S_{1} \mathbf{I} & S_{2} \mathbf{I} & S_{3} \mathbf{I} & S_{4} \mathbf{I} & S_{5} \mathbf{I} & S_{6} \mathbf{I} & S_{7} \mathbf{I} & S_{8} \mathbf{I}
\end{array}\right]
$$

where $\mathbf{I}$ is $3 \times 3$ identity matrix and $S_{i}=S(\xi, \eta, \zeta)$ and its values can be obtained by local coordinates $x, y, z$ and defined as

$$
\begin{aligned}
& S_{1}=1-3 \xi^{2}+2 \xi^{3} \\
& S_{2}=l_{e}\left(\xi-2 \xi^{2}+\xi^{3}\right) \\
& S_{3}=l_{e}(\eta-\xi \eta) \\
& S_{4}=l_{e}(\zeta-\xi \zeta) \\
& S_{5}=3 \xi^{2}-2 \xi^{3} \\
& S_{6}=l_{e}\left(2 \xi^{2}+\xi^{3}\right) \\
& S_{7}=l_{e} \xi \eta \\
& S_{8}=l_{e} \xi \zeta
\end{aligned}
$$

The nondimensional quantities $\xi, \eta, \zeta$ are defined as

$$
\begin{aligned}
\xi & =\frac{x}{l_{e}} \\
\eta & =\frac{y}{l_{e}} \\
\zeta & =\frac{z}{l_{e}}
\end{aligned}
$$

and $l_{e}$ is the length of the yarn beam element in the undeformed configuration (straight configuration, also see Figure 4).

Since the cross-section of the element of the moving yarn segment is assumed to be circular, for the convenience of calculation, the following coordinate transformation can be performed, as shown in Figure 3. Therefore, the global element shape function matrix $\mathbf{S}(x, y, z)$ and be transformed into $\mathbf{S}(x, r, \theta)$ :

$$
\begin{aligned}
& y=r \cos \theta \\
& z=r \sin \theta \\
& x \in\left[0, l_{e}\right], \\
& r \in\left[0, \frac{d}{2}\right], \\
& \theta \in[-\pi, \pi] .
\end{aligned}
$$

where $d$ is the equivalent diameter of the cross-section of the yarn segment. The generalized mass matrix of the moving yarn segment element in the ANCF is a constant matrix [25]:

$$
\mathbf{M}=\int_{V} \rho_{\text {yarn }} \mathbf{S}^{\mathrm{T}} \mathbf{S} \cdot \mathrm{d} V
$$

where $\rho_{\text {yarn }}$ is the linear mass density of the yarn. 


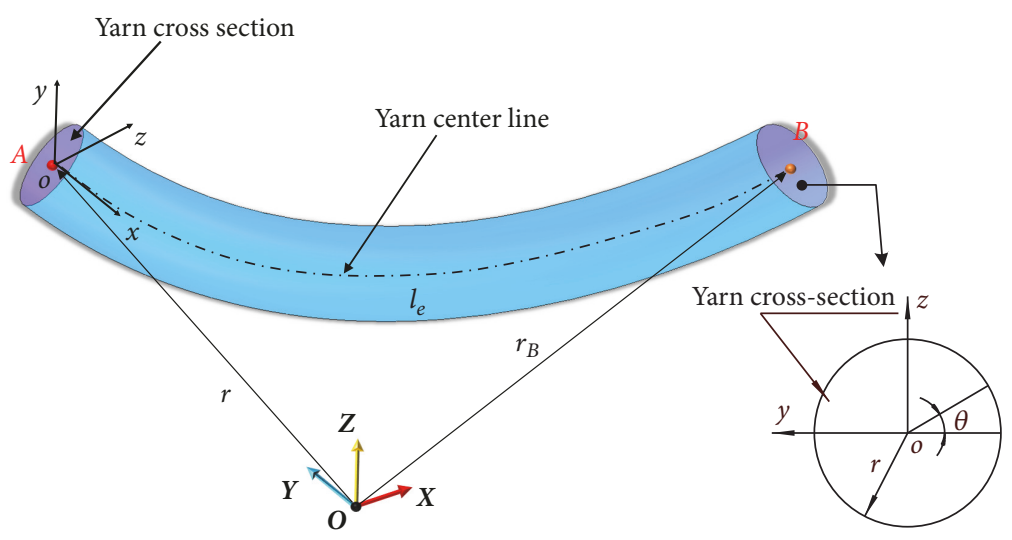

FIGURE 3: The moving yarn segment element model.

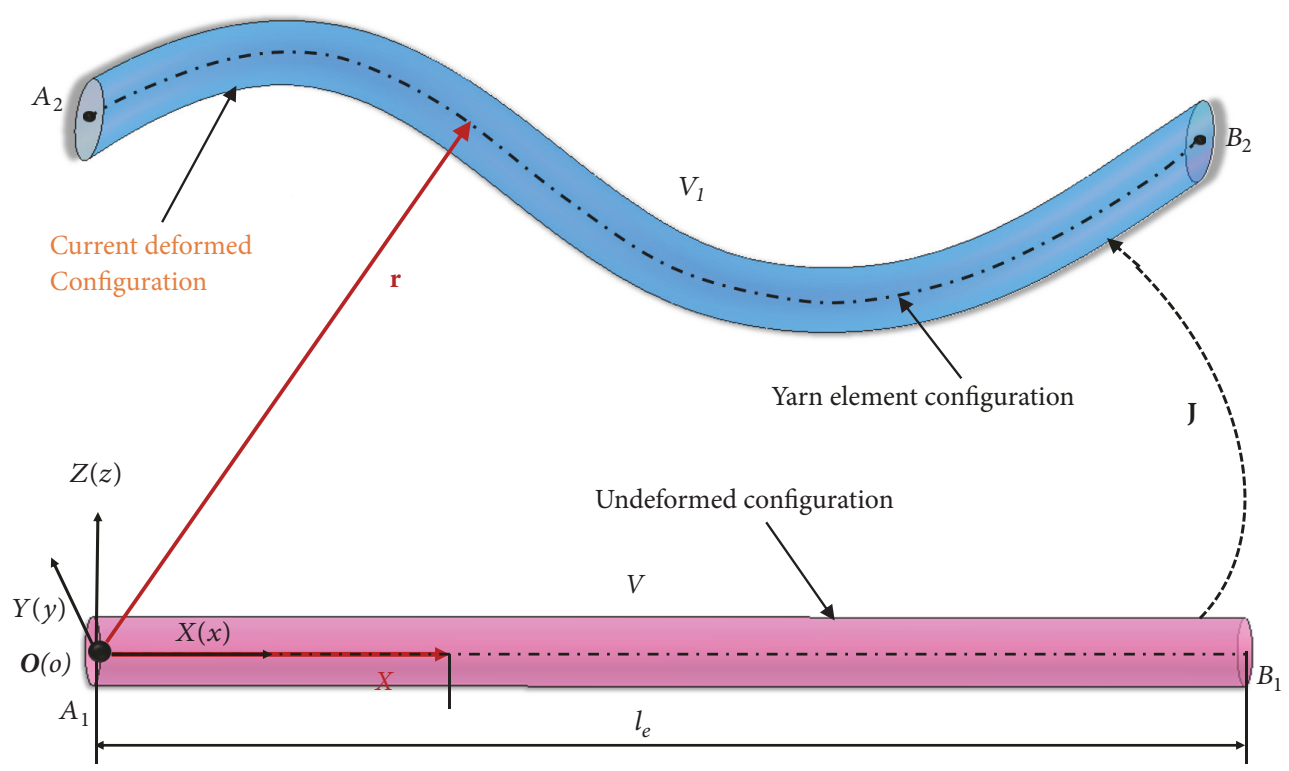

FIGURE 4: Configurations of moving yarn.

2.1.3. Kinematics and Deformation. Based on continuum mechanics, there are two kinds of configurations that are used to describe the deformation and motion of the moving yarn segment, as shown in Figure 4.

The two configurations can be defined in the global coordinate system $O X Y Z$, which are called the undeformed configuration and the current deformed configuration. The undeformed configuration describes the moving yarn segment element, which is in a straight condition, as well as a reference object. Its local coordinate system oxyz coincides with the global coordinate system $O X Y Z$, and the volume of the element is $V$. After a period of time, the moving yarn segment will move and deform, and the element will be in the current deformed configuration; the coordinates of the yarn element in the current configuration is expressed as $\mathbf{r}$ and its volume as $V_{1}$.

The kinetic energy of a yarn element can be defined as

$$
\mathbf{T}=\frac{1}{2} \int_{\mathrm{V}} \rho_{\text {yarn }} \dot{\mathbf{r}}^{\mathrm{T}} \dot{\mathbf{r}} \cdot \mathrm{dv}=\frac{1}{2} \dot{\mathbf{e}}^{\mathrm{T}} \mathbf{M} \dot{\mathbf{e}}
$$

where $\rho_{\text {yarn }}$ is the density of the yarn.
2.1.4. Strain Energy. The element of the moving yarn segment will move and change from one configuration to another, which can be described by the Jacobian matrix $J$, which is a coordinate transformation matrix [16]. Based on the GreenLagrange strain and the second Piola-Kirchhoff tensor, the yarn's Green-Lagrange strain tensor $\varepsilon$ can be expressed as

$$
\boldsymbol{\varepsilon}=\left[\begin{array}{lll}
\varepsilon_{11} & \varepsilon_{12} & \varepsilon_{13} \\
& \varepsilon_{22} & \varepsilon_{23} \\
s y m & & \varepsilon_{33}
\end{array}\right]=\frac{1}{2}\left(\mathbf{J}^{\mathrm{T}} \mathbf{J}-\mathbf{I}\right)
$$

As the strain tensor is symmetric, six independent strain components can be identified that can be used to define the strain vector as

$$
\varepsilon_{\mathrm{v}}=\left[\begin{array}{llllll}
\varepsilon_{11} & \varepsilon_{22} & \varepsilon_{33} & \varepsilon_{12} & \varepsilon_{13} & \varepsilon_{23}
\end{array}\right]^{\mathrm{T}}
$$

where $\varepsilon_{i j}$, for $i=j$, are called normal strains and $\varepsilon_{i j}$, for $i \neq j$, are called shear strains. 
The stored strain energy density function in the element can be written as

$$
\mathbf{U}\left(\boldsymbol{\varepsilon}_{\mathrm{e}}+\boldsymbol{\varepsilon}_{\mathrm{vis}}\right)=\mathbf{U}_{\mathrm{e}}+\mathbf{D}_{\mathrm{vis}}
$$

where $\mathbf{U}_{e}$ denotes the elastic energy and $\mathbf{D}_{v i s}$ denotes the energy dissipated.

2.1.5. Generalized Elastic Forces. The elastic energy of the yarn element $\mathbf{U}_{e}$ can be written as

$$
\mathbf{U}_{\mathrm{e}}=\frac{1}{2} \int_{\mathrm{v}}\left(\mathbf{C}_{\mathrm{tr}} \varepsilon_{\mathrm{v}}\right)^{\mathrm{T}} \mathbf{E}\left(\mathbf{C}_{\mathrm{tr}} \varepsilon_{\mathrm{v}}\right) \cdot \mathrm{dv}
$$

where $\mathbf{E}$ is the fourth-order tensor of the matrix of the elastic coefficient that will be introduced in the following section: the constitutive equation of yarn. $\mathbf{C}_{t r}$ is a constant coefficient matrix and can be defined as follows:

$$
\mathbf{C}_{t r}=\left[\begin{array}{ll}
\mathbf{I} & 0 \\
0 & 2 \mathrm{I}
\end{array}\right]
$$

The elastic forces can be obtained as gradients of the elastic energy $\mathbf{U}_{e}$.

$$
\begin{aligned}
\mathbf{Q}_{\mathrm{e}}= & \frac{\partial \mathbf{U}_{\mathrm{e}}}{\partial \mathbf{e}} \\
= & \int_{\mathrm{v}}\left[\begin{array}{llllll}
\frac{\partial \varepsilon_{11}}{\partial \mathbf{e}} & \frac{\partial \varepsilon_{22}}{\partial \mathbf{e}} & \frac{\partial \varepsilon_{33}}{\partial \mathbf{e}} & 2 \frac{\partial \varepsilon_{12}}{\partial \mathbf{e}} & 2 \frac{\partial \varepsilon_{13}}{\partial \mathbf{e}} & 2 \frac{\partial \varepsilon_{23}}{\partial \mathbf{e}}
\end{array}\right] \cdot \mathbf{E} \\
& \bullet\left[\begin{array}{llllll}
\varepsilon_{11} & \varepsilon_{22} & \varepsilon_{33} & 2 \varepsilon_{12} & 2 \varepsilon_{13} & 2 \varepsilon_{23}
\end{array}\right]^{\mathrm{T}} \mathrm{dv}
\end{aligned}
$$

2.1.6. Generalized Viscos Force. The dissipated energy due to viscosity can be written as

$$
\mathbf{D}_{\text {vis }}=\frac{1}{2} \int_{\mathrm{v}} \eta_{\mathrm{vis}}\left(\dot{\boldsymbol{\varepsilon}}_{\mathrm{vis}}\right)^{2} \mathrm{dv}
$$

where $\eta_{\text {vis }}$ is the viscosity coefficient of the yarn element and $\dot{\boldsymbol{\varepsilon}}_{\text {vis }}$ is the viscous strain rate. $\boldsymbol{\varepsilon}_{\text {vis }}$ is the viscous strain, which is governed by the following equations:

$$
\dot{\boldsymbol{\varepsilon}}_{v i s}+\frac{1}{\tau} \boldsymbol{\varepsilon}_{v i s}=\frac{1}{\tau} \boldsymbol{\varepsilon}
$$

where $\tau$ is called the relaxation time, $\tau=\eta_{\text {vis }} / E_{\mathrm{L}}$, and where $E_{\mathrm{L}}$ is the tensile elastic modulus in the longitudinal direction.

The generalized viscosity force can then be written as

$$
\mathbf{Q}_{\mathrm{vis}}=\frac{\partial \mathbf{D}_{\mathrm{vis}}}{\partial \mathbf{e}}
$$

2.1.7. Generalized Gravity. When the yarn is moving in the processing space, the yarn is subjected to the force due to gravity. If the principle of conservation of mass or the continuity condition is assumed, the virtual work of the inertial force can be written as

$$
\mathbf{W}_{\mathrm{i}}=\int_{\mathrm{v}_{1}} \rho_{\mathrm{yarn}} \mathbf{a}^{\mathrm{T}} \delta \operatorname{rdv}_{1}
$$

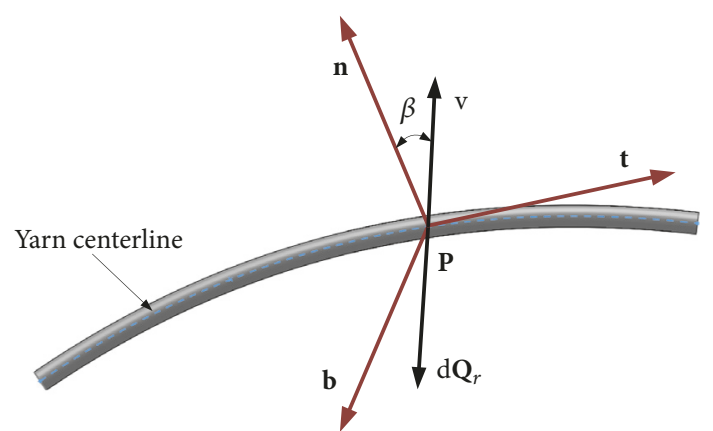

FIGURE 5: Air resistance of the moving yarn.

where $\mathrm{v}_{1}$ is the volume of the yarn in the current configuration, $\mathrm{v}_{1}=|\mathrm{J}| \cdot \mathrm{d} v, \rho_{\text {yarn }}$ is the density of the yarn, and a is the vector due to gravitational acceleration in the global coordinate system. If the gravitational acceleration in the direction of $-\mathrm{Z}$ is defined, then the vector a can be written as $\mathbf{a}=\left[\begin{array}{ll}0 & 0-g\end{array}\right]^{\mathrm{T}}$, where $g$ is the acceleration due to gravity.

From formula (18), the generalized gravity force formula can be obtained and written as

$$
\mathbf{Q}_{\mathrm{g}}=\frac{\delta \mathbf{W}_{\mathrm{i}}}{\delta \mathbf{e}}
$$

2.1.8. Air Resistance Forces. Apart from the body forces, the yarn is subject to surface forces such as air resistance, which plays a vital role during the movement of the yarn. Figure 5 shows a side view of a yarn that is subject to air resistance.

The vectors $\mathbf{b}, \mathbf{n}, \mathbf{t}$ and are three sets of mutually perpendicular vectors. $\mathbf{t}$ is the tangent to the centerline of the yarn at point $P, \mathbf{n}$ is perpendicular to the centerline, and $\mathbf{b}$ is the binormal vector. The three vectors have the relationship:

$$
\begin{aligned}
& \mathbf{b}=\frac{\boldsymbol{r}^{\prime} \times \boldsymbol{r}^{\prime \prime}}{\left\|\boldsymbol{r}^{\prime} \times \boldsymbol{r}^{\prime \prime}\right\|} \\
& \mathbf{n}=\mathbf{b} \times \mathbf{t}=\frac{\left(\boldsymbol{r}^{\prime} \times \boldsymbol{r}^{\prime \prime}\right) \times \boldsymbol{r}^{\prime}}{\left\|\boldsymbol{r}^{\prime} \times \boldsymbol{r}^{\prime \prime}\right\| \bullet\left\|\boldsymbol{r}^{\prime}\right\|}
\end{aligned}
$$

When the yarn is moving with a given velocity $\mathbf{v}$, the air resistance force is acting against $\mathbf{v}$ in accordance with the formula:

$$
\mathrm{d} \mathbf{Q}_{\mathrm{r}}=\frac{1}{2} C_{\mathrm{r}} \rho_{\text {air }} \mathrm{dA} \cos \beta\|\mathbf{v}\| \mathbf{v}
$$

where $C_{\mathrm{r}}=0.34$, which is the drag coefficient of a streamlined body [26]. $\rho_{\text {air }}$ is the density of the air. dA $\cos \beta$ is the effective area of the cross section of yarn. $\beta$ is the angle between the vectors $\mathbf{v}$ and $\mathbf{n}$.

The exact formula of the air resistance of the yarn element can be written as

$$
\mathbf{Q}_{\mathrm{r}}=\int_{\mathrm{V}} \frac{1}{2} C_{\mathrm{r}} \rho_{\text {air }} \mathrm{dA} \cos \beta\|\mathbf{v}\| \mathbf{v d V}
$$

2.1.9. Dynamics Equations. Under the action of gravity $\mathbf{Q}_{g}$, the elastic force $\mathbf{Q}_{e}$, and the generalized viscosity force due 
to deformation and the air resistance force $\mathbf{Q}_{r}$, the dynamics equation of the yarn can be expressed by the generalized force as

$$
\mathbf{M e ̈}(x, y, z, t)+\mathbf{Q}_{\mathrm{e}}+\mathbf{Q}_{\mathrm{vis}}=\mathbf{Q}_{\mathrm{g}}+\mathbf{Q}_{\mathrm{r}}
$$

2.2. Constitutive Equation. In this section, the constitutive model of the yarn has been discussed. The yarn, which is the fiber assembly, has a special structure in comparison to traditional materials. In the existing research, and, depending on the state of the yarn, there have been different descriptions of the yarn: pure elasticity, viscosity, and viscoelasticity. For instance, when discussing the coupling of the fiber and the airflow [27-29], especially during vortex spinning, the fibers or yarns were considered as a pure elastic body in either one or two dimensions. When analyzing the vibration performance of the moving yarn, it was treated as a onedimensional viscoelastic body [30]. These studies ignored the coupling between stretching, shearing, and torsion of the yarn, which resulted in an inaccurate description of the yarn and produced large deformation and a wide range of movement. When the yarn was moving it displayed elasticity while also exhibiting some viscosity. Therefore, in order to ensure that the elastic characteristics of the yarn are fully described in all directions and, at the same time, that the viscoelastic characteristics are also described in the longitudinal direction, that is, the main direction in which the yarn is subjected to tension during the manufacturing process, and the following method has been used in this paper to calculate the constitutive relationship of the yarn.

Therefore, the total strain energy of the yarn consists of two parts, the first is the elastic strain energy, and the second is the dissipation energy. Correspondingly, the stress can be written as

$$
\sigma=\sigma_{\mathrm{e}}+\sigma_{\text {vis }}
$$

where $\sigma_{\mathrm{e}}$ denotes the elastic force and $\sigma_{\text {vis }}$ represents the viscosity force. The strain and stress of the yarn can be linked using the constitutive relation. In the past 30 years, many scholars have studied the equivalent constitutive relation of yarn $[17,31,32]$. In order to simplify the description as well as the modeling of the yarn, it can also be considered as a material that does not possess structural parameters, ignoring the effects between single fibers in the yarn. Since the yarn can be considered as isotropic and symmetrical in cross-section, transversely isotropic elastomers can be used to describe the elastic performance of the yarn. The relationship between stress and strain can be written as follows:

$$
\left\{\begin{array}{l}
\varepsilon_{\mathrm{e}_{\mathrm{xx}}} \\
\varepsilon_{\mathrm{e}_{\mathrm{yy}}} \\
\varepsilon_{\mathrm{e}_{\mathrm{zz}}} \\
\varepsilon_{\mathrm{e}_{\mathrm{ly}}} \\
\varepsilon_{\mathrm{e}_{\mathrm{zl}}} \\
\varepsilon_{\mathrm{e}_{\mathrm{yz}}}
\end{array}\right\}=\mathrm{C}\left\{\begin{array}{c}
\sigma_{\mathrm{e}_{\mathrm{x}}} \\
\sigma_{\mathrm{e}_{\mathrm{y}}} \\
\sigma_{\mathrm{e}_{\mathrm{z}}} \\
\sigma_{\mathrm{e}_{\mathrm{xy}}} \\
\sigma_{\mathrm{e}_{\mathrm{zx}}} \\
\sigma_{\mathrm{e}_{\mathrm{yz}}}
\end{array}\right\}
$$

where $\varepsilon_{\mathrm{e}_{\mathrm{ij}}}, i, j=x, y, z$ denote the axial and shear GreenLagrange strains and $\mathbf{C}$ is the matrix of the elastic coefficients.
When a yarn is defined as having square-symmetric and transversely isotropic homogeneous continuum, the elastic compliance matrix can be expressed by the modulus and Poisson's ratios [33]:

$$
\mathbf{C}=\left[\begin{array}{cccccc}
\frac{1}{E_{L}} & -\frac{v_{T L}}{E_{T}} & -\frac{v_{T L}}{E_{T}} & 0 & 0 & 0 \\
-\frac{v_{L T}}{E_{L}} & \frac{1}{E_{T}} & -\frac{v_{T T}}{E_{T}} & 0 & 0 & 0 \\
-\frac{v_{L T}}{E_{L}} & -\frac{v_{T T}}{E_{T}} & \frac{1}{E_{T}} & 0 & 0 & 0 \\
0 & 0 & 0 & \frac{1}{G_{T L}} & 0 & 0 \\
0 & 0 & 0 & 0 & \frac{1}{G_{T L}} & 0 \\
0 & 0 & 0 & 0 & 0 & \frac{1}{G_{T T}}
\end{array}\right]
$$

where $E_{L}$ is the longitudinal elastic modulus governing the uniaxial loading in the longitudinal direction (along the $x$ axis of the yarn); $v_{L T}$ is the associated Poisson's ratio that accounts for the effect of the strains in the transverse directions; $E_{T}$ is the transverse elastic modulus (in the crosssection, $y$ or $z$ direction); $v_{T T}$ is the associated Poisson's ratio governing the resultant strains in the remaining orthogonal transverse directions ( $y$ or $z$ direction); $v_{T L}$ is corresponding Poisson's ratio governing the induced strain in the longitudinal direction, $G_{T L}$ is the shear modulus governing shear in the longitudinal direction, and $G_{T T}$ is the shear modulus governing shear in the transverse plane.

In the case of transverse isotropy, $G_{T T}$ is a dependent modulus which is equal to $G_{T T}=E_{T} / 2\left(1+v_{T T}\right)$, and $G_{T L}=$ $E_{T} / 2\left(1+v_{T L}\right)$.

The yarn also exhibits viscosity during large deformation and movement. In particular, the yarn has a damping effect of the nonnegligible viscous force in the longitudinal direction due to its tension. Moreover, since the yarn has a sizeable tensile modulus in the longitudinal direction, it also exhibits a particular form of oscillation during movement, which further indicates that the energy dissipated by the viscous force in the longitudinal direction cannot be ignored. When considering the viscosity of the yarn in the longitudinal direction, the stress-strain relationship can be written as follows:

$$
\sigma_{\mathrm{vis}}=\eta_{\mathrm{vis}} \frac{\mathrm{d} \varepsilon_{x x}}{\mathrm{~d} t}
$$

where $\eta_{\text {vis }}$ is usually measured through static testing and $\mathrm{d} \varepsilon_{x x} / \mathrm{d} t$ is the strain rate of the yarn in the longitudinal direction. This strain is the same as the strain in the longitudinal direction when calculating the elastic strain energy. This also indicates that the description of the viscoelasticity and elasticity in the longitudinal direction are interrelated.

2.3. Solution Procedure. In this study, the governing equation for the yarn segment motion Equation (24) is solved using the ODE45 method to obtain the global position vectors of each node. The solution procedure which is shown in Figure 6 is as follows: 


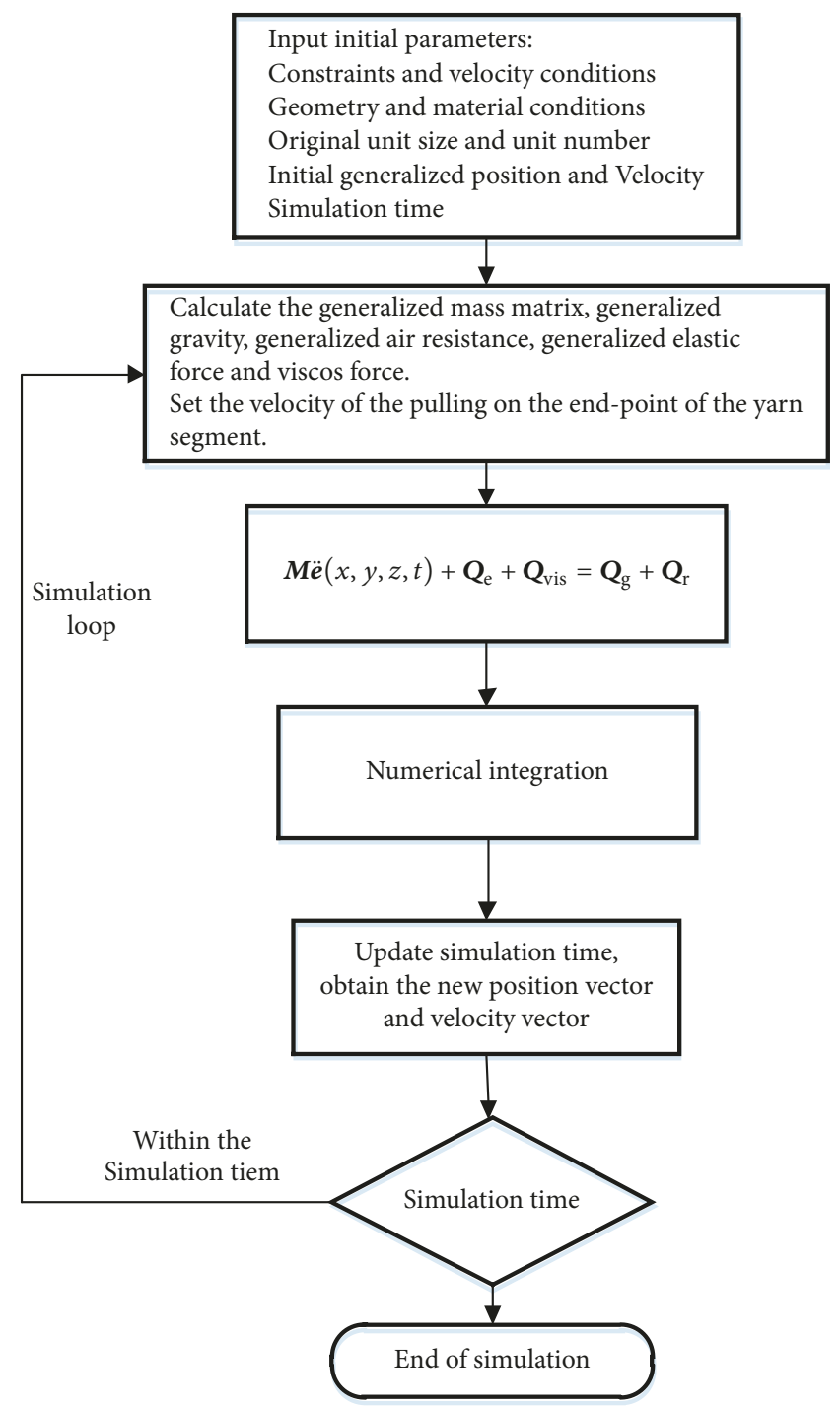

FIGURE 6: Solution procedure.

(1) Set the calculation parameters including simulation time. Set the geometrical and physical parameters of the yarn segment.

(2) Set the initial configuration of the yarn segment, including the generalized initial position and generalized initial velocity of the yarn segment.

(3) Set the element number of the model.

(4) Set the velocity of one endpoint of the yarn segment, which is the yarn guider's velocity.

(5) Calculate the generalized mass matrix and generalized elastic force, viscos force, gravity, and air resistance force.

(6) Solve the governing equations for the yarn segment motion to obtain the new position vector and velocity vector.

(7) Return to step (4) and repeat the procedure until the end of the computation is reached.

\section{Experimental Validation}

3.1. Motions of Pulling the Yarn Segment in the Air. In this section, the results of the simulation and the experiments with a moving yarn segment have been presented. The results have been shown here to demonstrate the effectiveness of the proposed moving yarn segment model. For this reason, a polyester filament bundle, which is the most basic type of the yarn, was used as the object for the simulations and experiments. As the cross-section of the polyester monofilament is circular and the bundle of filaments is approximately parallel when arranged in the straightened state, this is more in line with the assumption that was made for the constitutive relationship.

3.1.1. The Initial Configuration and Movement. Figure 7 shows the initial position that was assumed before the simulation was carried out. The traction fixer held the endpoint of the filament bundle. For the specific clamping method see the section described in "Clamping Mode", and the other endpoint was clamped in the same way. The initial filament bundle remained horizontal, but the tension was nearly zero, which was ensured using the precision tension measuring instrument. At the beginning of the experiment, the traction 


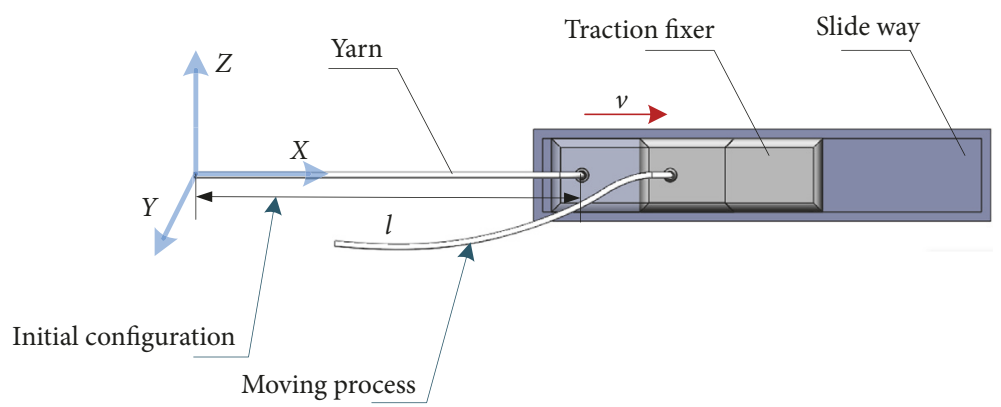

FIGURE 7: Moving yarn model for simulation and experiment.

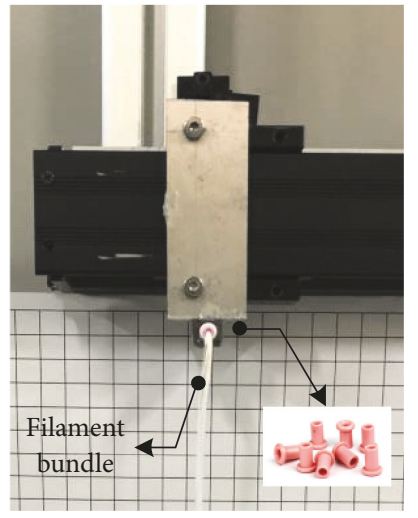

FIGURE 8: Clamped filament bundle.

fixer propelled the end of the yarn and moved it at a constant speed, while the constraint at the other end was released, and the filament bundle was pulled down by gravity. The trajectory of the filament bundle was recorded using a highspeed camera.

3.1.2. Clamping Mode. Firstly, the clamped end of the filament bundle was fixed with a porcelain eye that is commonly used to guide yarns in the process. The porcelain eye is a ceramic workpiece with a diameter of $0.5 \mathrm{~mm}$, as shown in Figure 8 , so that the cross-section of the restrained end of the filament bundle is guaranteed to be a circle. The filament bundle is wetted by an oil agent so that the monofilaments are tightly held together, and the middle section of the filament bundle can also be considered to be approximately circular. The filament bundle is fixed to the porcelain eye by sticky glue. In order to ensure that the yarn was not contaminated by the glue, the fixed section of the filament bundle and the porcelain eye were not included in the sample that was used in the experiment. The porcelain eye was mounted on the clamping plate with the traction fixer, as shown in Figure 8.

3.1.3. Traction Speed and Total Stroke. The purpose of the simulation and the experiment was to verify the accuracy of the moving yarn segment model. Since the length of the simulated filament bundle was $0.32 \mathrm{~mm}$, in order to observe the trajectory of the movement more clearly, it needed to be moved at least $1 / 3$ of its length during the experiment.
TABLE 1: The parameters of the filament bundle used in simulation and experiment.

\begin{tabular}{lc}
\hline Parameter & Value and unit \\
\hline The density of the polyester material & $1320 \mathrm{~kg} / \mathrm{m}^{3}$ \\
Linear mass density (measured value) & $614.2 \mathrm{tex}$ \\
Tensile modulus & $1.03 \times 10^{8} \mathrm{~N} / \mathrm{m}^{2}$ \\
Viscosity coefficient & $3.7 \times 10^{8} \mathrm{~N} \cdot \mathrm{s} / \mathrm{m}^{2}$ \\
Fiber volume fraction & $70.16 \%$ \\
Length & $320 \mathrm{~mm}$ \\
Cross section diameter & $0.92 \mathrm{~mm}$ \\
Air density & $1.2 \mathrm{~kg} / \mathrm{m}^{3}$ \\
\hline
\end{tabular}

Combined with the simulation time and the data store capacity of the high-speed camera, the speed of the traction motion was determined to be $0.1 \mathrm{~m} / \mathrm{s}$, according to the stroke length.

3.1.4. Determination of Physical Parameters. The POY filament bundle had a nominal denier of 220dtex/72f. For better observation, 24 bundles of these POY filaments were combined into a bundle. Therefore, the actual linear mass density of the filament bundle was 5280dtex. A precision length measuring instrument (YG086) used to measure the length of the sample which was $320 \mathrm{~mm}$. According to the product manual, the density of the polyester was $1.38 \mathrm{~g} / \mathrm{m}^{3}$. Before the experiment, the actual linear mass density of the filament bundle was measured using a precision balance (PRACTUM2102-1CN) according to the standard method of testing linear mass density [34]. The actual diameter of the filament bundle was measured using scanning electron microscopy (JCM-6000PLUS).

3.1.5. Determination of Constitutive Parameters. The tensile modulus of the filament bundle was measured using a large-scale single yarn strength gauge (SITHAI ST-D100). According to the assumed idealized conditions, the shear modulus and Poisson's ratio were determined using equation $=E / 2(1+v)$.

The modulus and Poisson's ratio in the other directions could be determined using the method in the literature [33]. The viscosity coefficient used the parameters that were found in the literature [30]. Table 1 lists the parameters of the 


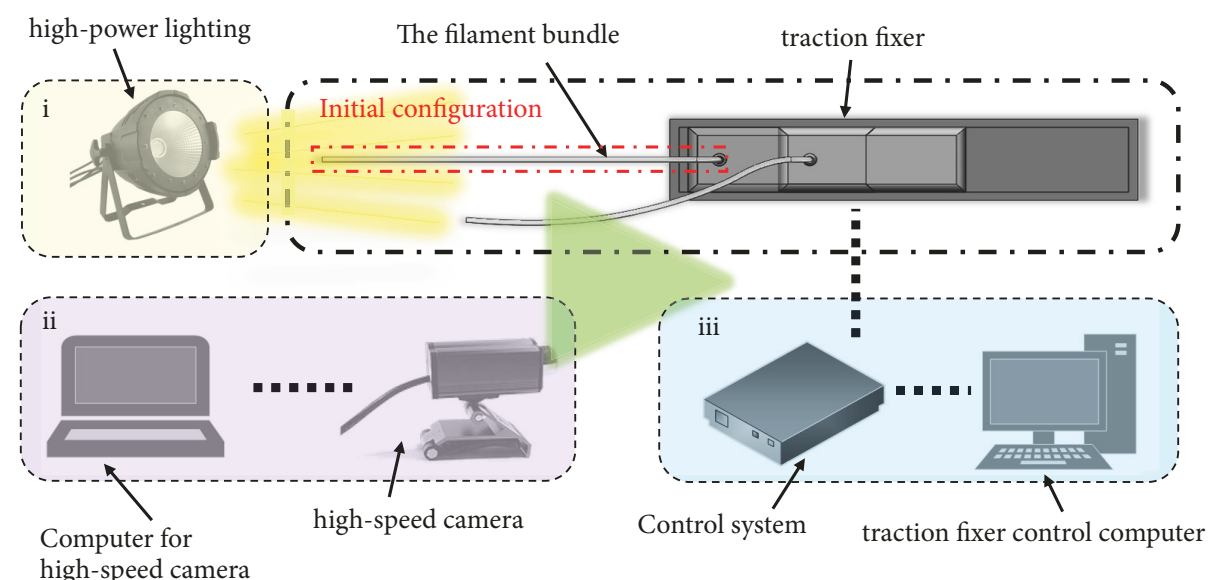

(a)

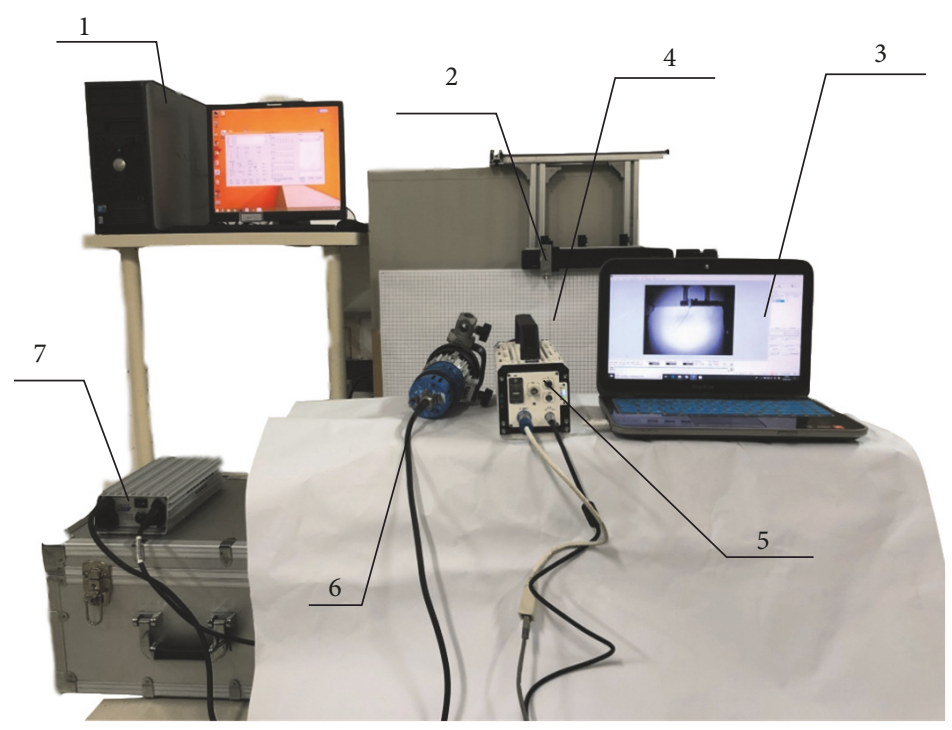

(b)

FIGURE 9: Moving yarn model for simulation and experiment. (a) schematic diagram and (b) test site. (1) Traction fixer control computer; (2) traction fixer; (3) computer for high-speed camera; (4) the filament bundle; (5) high-speed camera; (6) high-power lighting; (7) control system.

filament bundle that were used in the simulation. Figures 9(a) and 9 (b) show the experiment schematic diagram and test site picture.

3.2. Results. When the traction fixer, which clamped the endpoint of the filament bundle, began to move, the other endpoint was released. A high-speed camera recorded the moving trace of the filament bundle, which was used to validate the model of the yarn. The motion states of the filament bundle at $0.05,0.1$, and $0.6 \mathrm{~s}$ were shown in Figures 10 (a) $-10(\mathrm{c})$. The comparison of the results has been shown in Figure 10(d).

The filament bundle moved under the pulling action of the fixer. Figures 10 have shown that the positions of the filament bundle, for the different times that were obtained from the simulation and the experimental test, were very similar.
The moving trace of the filament bundle at $0.6 \mathrm{~s}$ was almost the same but they diverged at $0.25 \mathrm{~s}$ especially in the middle section. The experimental results showed a more curved result than that from the simulated results. The reasons for the difference were most likely as follows: (a) the motion of the filament bundle was affected by the air resistance. Thus, the appropriate air resistance coefficient should be incorporated into the simulation. The simulation in this paper used the results from the literature [26]. (b) The viscoelasticity of the filament bundle has a very significant effect on the results. The simulation in this paper only considered the viscosity in the longitudinal direction, which is a reasonable and efficient assumption when the moving yarn segment is subjected to high tension. Otherwise, the three-dimensional viscosity should be taken into consideration during the modeling. 

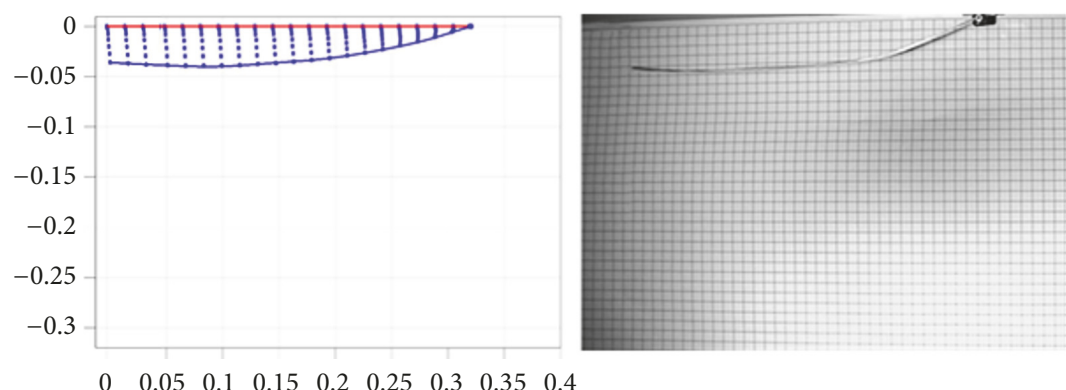

(a)
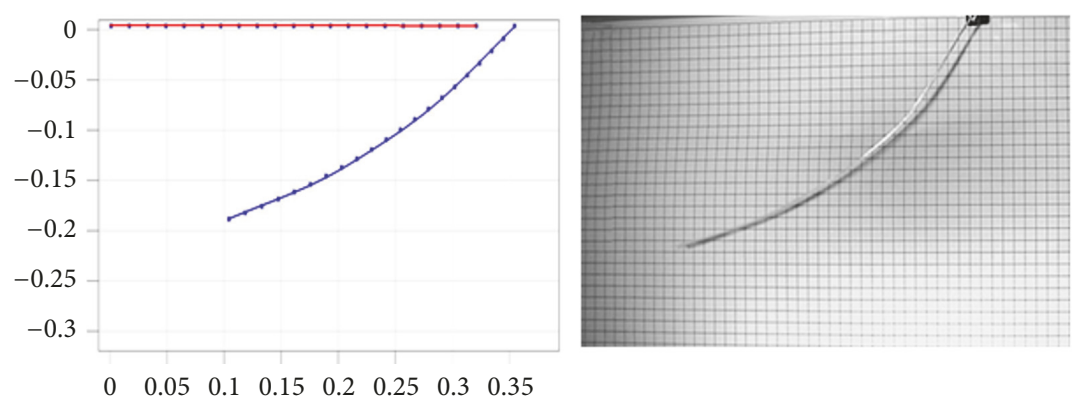

(b)
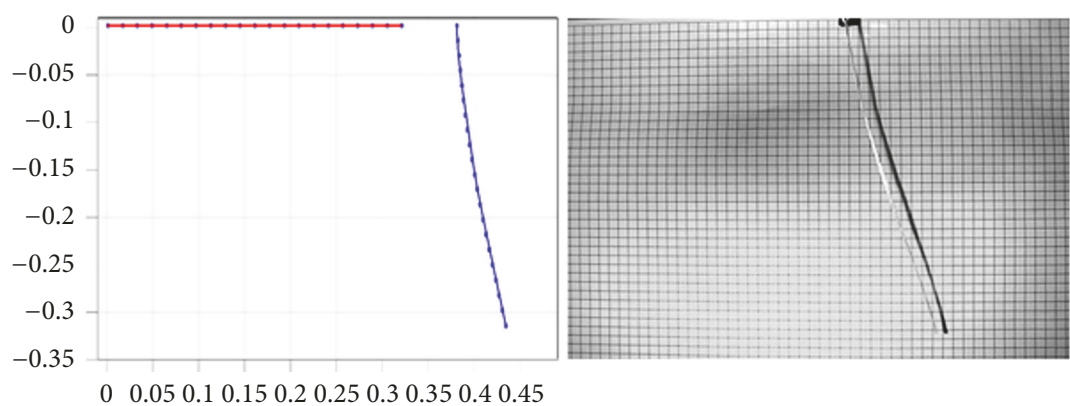

(c)

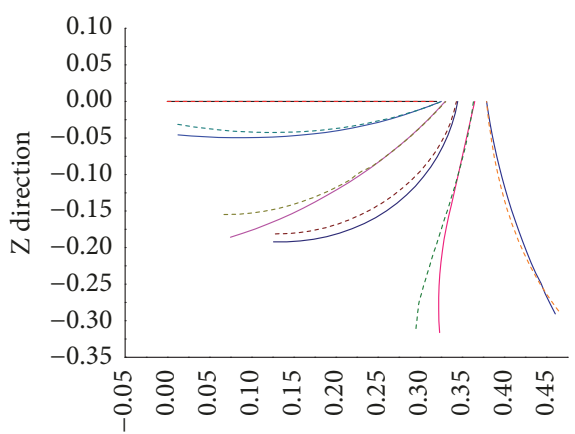

$\mathrm{X}$ direction

$$
\begin{aligned}
& \text { - } \mathrm{t}=0 \text {, simulation } \quad-\mathrm{t}=0.255 \text {, simulation } \\
& \text {-.- } \mathrm{t}=0 \text {, experiment } \quad \ldots \mathrm{t}=0.255 \text {, experiment } \\
& \text { - } \mathrm{t}=0.05 \text {, simulation }-\mathrm{t}=0.45 \text {, simulation } \\
& \text {-. } \mathrm{t}=0.05 \text {, experiment } \ldots \mathrm{t}=0.45 \text {, experiment } \\
& \text { - } \mathrm{t}=0.1 \text {, simulation } \quad-\mathrm{t}=0.6 \text {, simulation } \\
& \ldots \mathrm{t}=0.1 \text {,experiment } \ldots \mathrm{t}=0.6 \text {,experiment }
\end{aligned}
$$

(d)

FIgURE 10: Experimental validation. (a) Motion comparison at 0.05s. (b) Motion comparison of 0.1s. (c) Motion comparison of 0.6s. (d) Experimental and simulation comparison. 


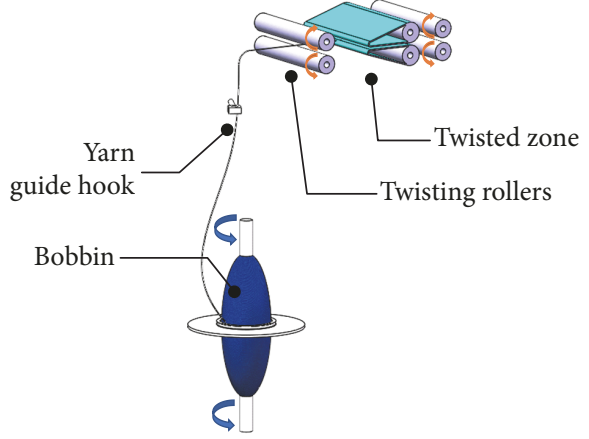

FIGURE 11: Yarn spinning process.

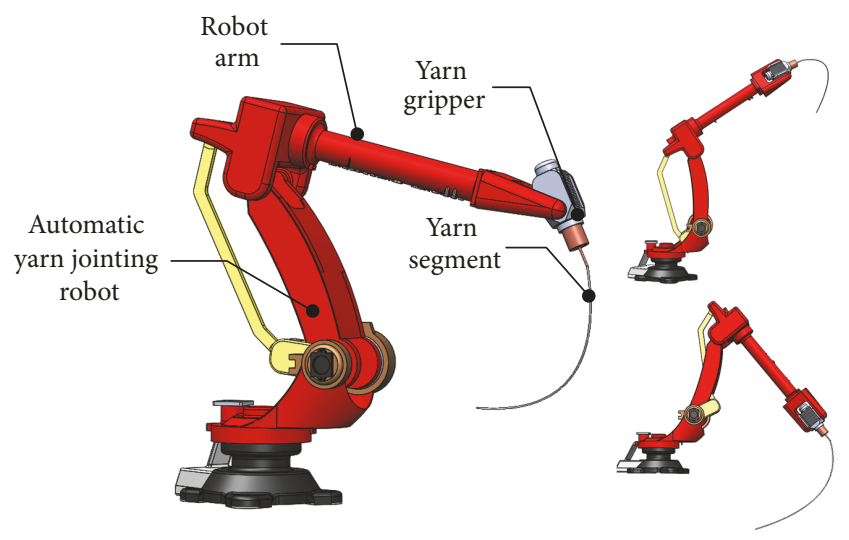

FIGURE 12: Automatic yarn-jointing robot.

\section{Motion of the Yarn Segment during the Automatic Yarn Piecing Process}

Automatic yarn piecing technology is the key technology needed to realize the intelligent spinning process. The development of an automatic piecing robot would play a significant role in enhancing the yarn spinning efficiency and guaranteeing the spinning quality. Already, relatively mature automatic piecing devices have been developed such as the FIL-A-MAT automatic piecing device (based on the Zinser 320 spinning machine), Fiasol automatic piecing device (Came Italy), Heathpan automatic piecing device, AYPT type automatic piecing device, and ROBOFIL automatic piecing device, and these devices can usually complete the detecting and piecing up process in less than 30 seconds. While developing automatic piecing technology, the design and control of the yarn piecing up module are major concerns. The yarn segment is pulled through the yarn guide hook and fed into the twist zone during this process. Finding the best method to complete the yarn piecing action quickly and accurately is the primary issue.

Taking the working process of the automatic yarn piecing robot during ring spinning as an example, the automatic piecing up work is performed by a robot arm. Figure 11 presents a schematic view of the ring spinning process. The robot arm must pull the yarn segment (Figure 12) to complete a series of actions such as winding it onto the bobbin, passing
TABLE 2: The parameters of the cotton yarn used in the simulation.

\begin{tabular}{lc}
\hline Parameter & Value and unit \\
\hline The density of the cotton yarn & $1.5 \times 10^{3} \mathrm{~kg} / \mathrm{m}^{3}$ \\
Linear mass density (measured value) & $324.2 \mathrm{tex}$ \\
Tensile modulus & $2.43 \times 10^{8} \mathrm{~N} / \mathrm{m}^{2}$ \\
Viscosity coefficient & $1.52 \times 10^{8} \mathrm{~N} \cdot \mathrm{s} / \mathrm{m}^{2}$ \\
Fiber volume fraction & $69.55 \%$ \\
Length & $320 \mathrm{~mm}$ \\
Cross section diameter & $0.66 \mathrm{~mm}$ \\
Air density & $1.2 \mathrm{~kg} / \mathrm{m}^{3}$ \\
\hline
\end{tabular}

it through the yarn guide hook and feeding it into the twisting rollers, as shown in Figure 13. Whether the yarn segment can complete the actions mentioned above under the guidance of the robot arm will determine the results of the automatic yarn piecing. Thus, predicting the trajectory of the yarn segment for different pulling speeds can be used to design and control the motion planning of the robot arm. The simulation focused on the process of the cotton yarn segment passing through the yarn guide hook, to determine whether the designed velocity of the robot arm is suitable for pulling the yarn segment through the guide hook. Table 2 lists the parameters of the cotton yarn used in this simulation. The simulation results (Figure 14) showed the trajectory of the yarn segment being pulled by the robot arm gripper, which is under the designed velocity parameters, as shown in Table 3.

As shown in Table 2, the endpoint of the yarn segment was pulled by the robot arm at different velocities during the spatial motion. The simulation produced the trajectory of the yarn segment in the different positions, as shown in Figure 14. The yarn segment was gripped by the robot arm and first moved toward the yarn guide hook. The velocities in the $\mathrm{Y}$ and $\mathrm{Z}$ directions were both $1.5 \mathrm{~m} / \mathrm{s}$. To prevent the accidental occurrence of friction between the yarn segment and the guide hook support arm, after moving at the abovementioned speed for $0.08 \mathrm{~s}$, the robot arm changed the velocity and the direction of movement and pulled the yarn segment to move in the direction of the opening of the guide hook. After the yarn segment had passed through the opening of the yarn guide hook, the robot arm changed the direction and velocity of the movement again, and the yarn being moved in the opposite direction to the opening of the yarn guiding hook to ensure that the yarn segment was already inside the yarn guide hook. Figure 14(a) shows that when the robot arm gripper moved with the designed velocity, the yarn segment could move from the horizontal position to the center of the guide hook and did not come into contact with the support arm of the guide hook which is a requirement of the automatic yarn piecing up process. In Figure 14, yarns of different colors represent the trajectories of different yarn segments, and the three parts of Figures 14(b)-14(d) describe the change in the trajectory of yarn segments when they were moved at the three velocities mentioned above, as shown in Table 3 .

The case described above has shown that the model that has been introduced in this paper can predict the trajectory 
TABLE 3: The velocity of the robot arm (which gripped the endpoint of the yarn).

\begin{tabular}{|c|c|c|c|c|}
\hline $\begin{array}{l}\text { Position statement of the yarn } \\
\text { segment }\end{array}$ & $\begin{array}{l}\text { Velocity in the } Y \\
\text { direction }(\mathrm{m} / \mathrm{s})\end{array}$ & $\begin{array}{l}\text { Velocity in the } Z \\
\text { direction }(\mathrm{m} / \mathrm{s})\end{array}$ & $\begin{array}{l}\text { Simulation time/ moving } \\
\text { time (s) }\end{array}$ & $\begin{array}{c}\text { Corresponding } \\
\text { configuration in } \\
\text { Figure } 14\end{array}$ \\
\hline Straight position to position 1 & 1.5 & 1.5 & 0.08 & (b) \\
\hline Position 1 to position 2 & -4 & 1 & 0.05 & (c) \\
\hline Position 2 to position 3 & -2 & -0.4 & 0.05 & (d) \\
\hline
\end{tabular}

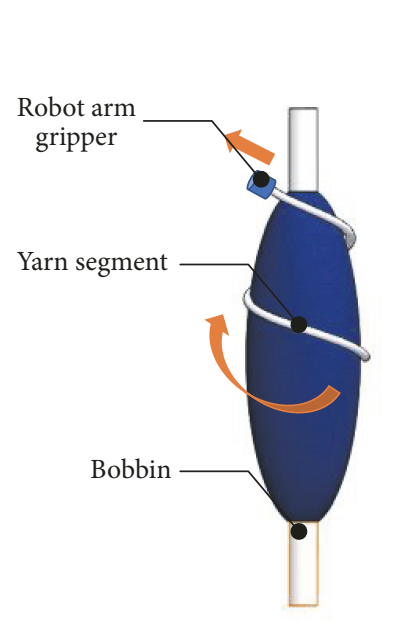

(a)

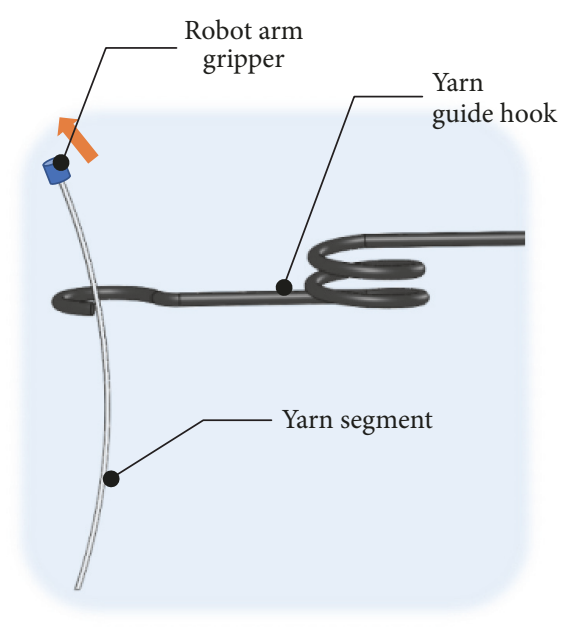

(b)

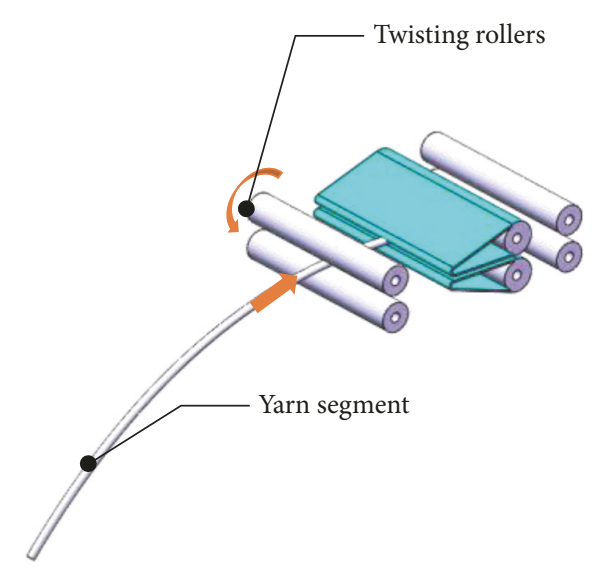

(c)

FIGURE 13: Yarn segment motion during the piecing up process by the robot arm. (a) Winding on the bobbin. (b) Passing through the yarn guide hook. (c) Feeding into the twisting rollers.

of the yarn segment under different pulling motions from the robot arm. On the basis of this model, and by modifying its boundary conditions or expanding the coupling condition of the yarn with the mechanical parts, it is now possible to predict other movement behaviors of the yarn segment of the automatic piecing process, such as winding the yarn onto the bobbin and feeding into the twisting zone.

\section{Conclusions}

This paper has proposed a dynamic model of a yarn segment to predict the yarn's motion trajectory based on the ANCF. When establishing the moving yarn segment model, the elasticity in the longitudinal direction and cross-section directions were considered, as well as the viscosity characteristic in the longitudinal direction. In order to enable the model to analyse the moving yarn segments for the actual processes, the effect of both air resistance and gravity were taken into consideration during the creation of the model.

As an example used to verify the simulation of the model, a model of the filament bundle where the gripper pulled it from one end on the guideway has been implemented this paper. The simulation and the corresponding experiment verified the accuracy of the model. Apart from this and in order to better understand how to use the model to predict the motion process of the moving yarn segment, another simulation was implemented which described the movement of the cotton yarn when it was pulled at different velocities during the automatic yarn piecing up process. This simulation described the yarn segment pulled from the horizontal position to the centre of the yarn guide hook. During this process, the yarn segment could not contact the support arm of the yarn guide hook. Thus, it was possible to check whether the designed velocity of the robot arm satisfies the requirements by observing the trajectory of the yarn segment.

On this basis, this model can be used effectively to provide a reference for the analysis of yarn segment motion during the textile process by extending the model with specific boundary conditions and constraints. Compared with modeling the yarn as either a moving string or a pure elastic body, the model that has been established in this paper is able to more effectively simulate the actual moving trace of the yarn segment when it is being pulled in the processes: such as the yarn segment protruding from the nozzle during air-jet weft insertion and the filament bundle segment during the start of the winding process. Through the use of the equations in this model, it is also possible to calculate the distribution of the tension in the yarn segment during movement. This has laid the groundwork for the establishment of a multibody system model for the coupling of the moving yarn segment with the mechanical part. For example, in order to simulate the dynamic behaviour of the yarn segment coupled to the gripper of the automatic yarn-jointing robot when in operation. Outlines for further research have been proposed as follows: 

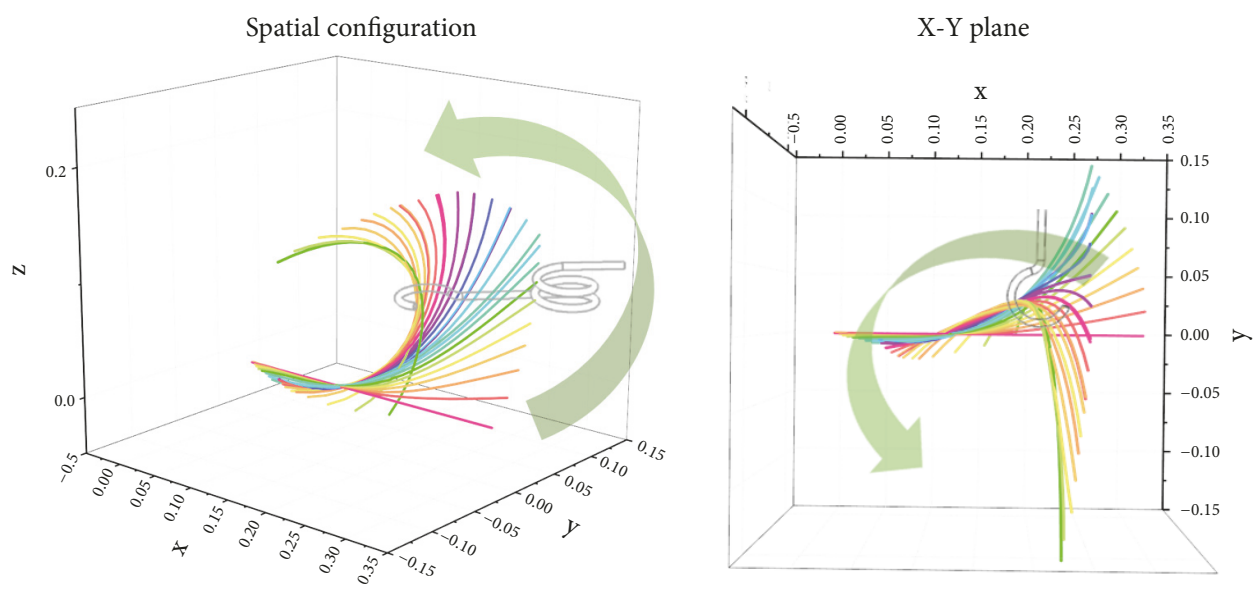

(a)

Spatial configuration

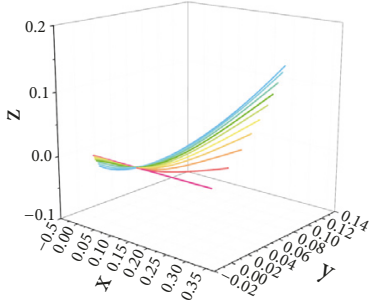

Spatial configuration

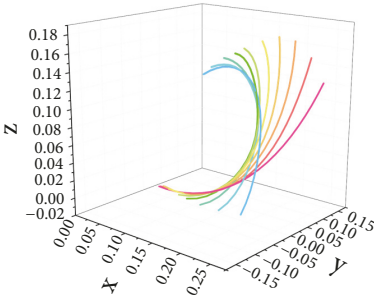

Spatial configuration

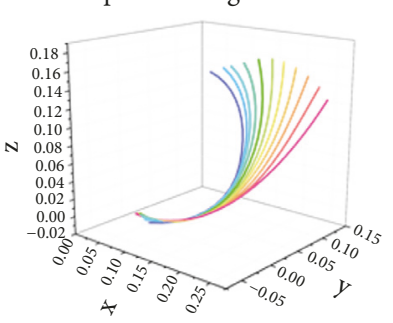

$\mathrm{X}-\mathrm{Y}$ plane

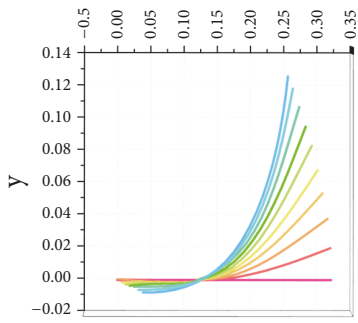

(b)

$\mathrm{X}-\mathrm{Z}$ plane

Y-Z plane
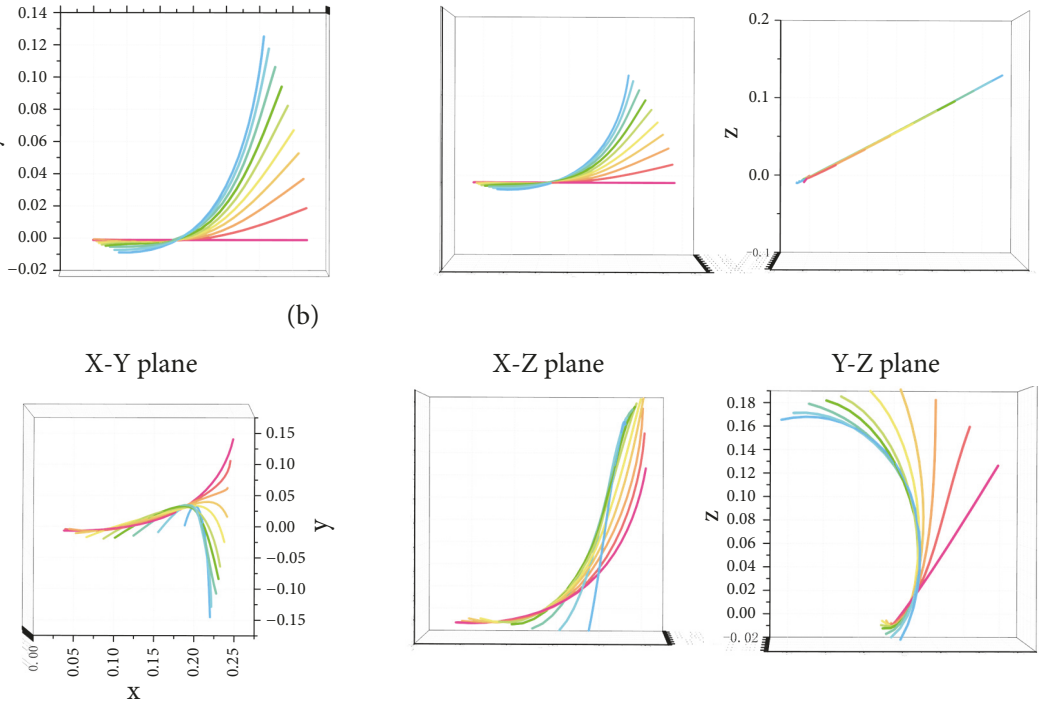

(c)

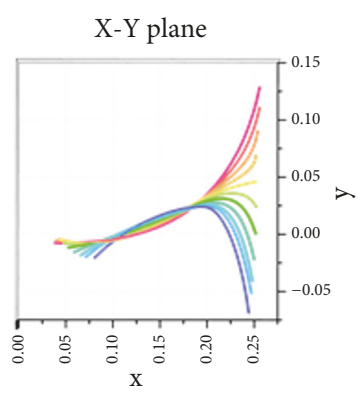

$\mathrm{X}-\mathrm{Z}$ plane

Y-Z plane
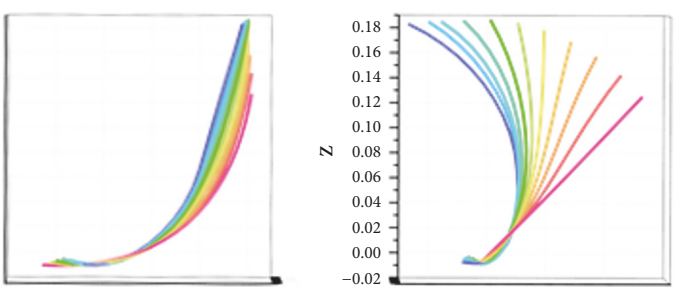

(d)

Figure 14: The spatial motion of the yarn segment under the pulling of robot arm. (a) The process of passing through the yarn guide hook. (b) The first position of the yarn segment. (c) The second position of the yarn segment. (d) The third position of the yarn segment.

(1) Accurate parameter identification is vital when dealing with the modeling of a specific yarn. Furthermore, the actual state of the yarn during the production process should be taken into consideration, such as its tension and temperature when determining the yarn's parameters.

(2) It is of interest to try to account for the viscosity of the cross-section dimension of the yarn, as well as using 
the three-dimension viscoelastic model to describe the constitutive relationship of the yarn. However, there is no doubt that this will also increase the amount of calculation inherent in the model.

\section{Data Availability}

The data used to support the findings of this study are available from the corresponding author upon request.

\section{Conflicts of Interest}

The authors declare no conflicts of interest.

\section{Acknowledgments}

This work was supported by the National Key R\&D Program of China (2017YFB1304000), Natural Science Foundation of Shanghai (16ZR1401900), and Applied Foundation Research of China National Textile and Apparel Council (J201504).

\section{References}

[1] Y. Zeng and C. Yu, "A bead-elastic rod model for dynamic simulation of fibers in high speed air flow," International Journal of Nonlinear Sciences and Numerical Simulation, vol. 4, no. 2, pp. 201-202, 2003.

[2] Y. Zeng and C. Yu, "Numerical simulation of fiber motion in the nozzle of an air-jet spinning machine," Textile Research Journal, vol. 74, no. 2, pp. 117-122, 2016.

[3] P. Skjetne, R. F. Ross, and D. J. Klingenberg, "Simulation of single fiber dynamics," The Journal of Chemical Physics, vol. 107, no. 6, pp. 2108-2121, 1997.

[4] S. Yamamoto and T. Matsuoka, "A method for dynamic simulation of rigid and flexible fibers in a flow field," The Journal of Chemical Physics, vol. 98, no. 1, pp. 644-650, 1993.

[5] R. F. Ross and D. J. Klingenberg, "Dynamic simulation of flexible fibers composed of linked rigid bodies," The Journal of Chemical Physics, vol. 106, no. 7, pp. 2949-2960, 1997.

[6] R. D. Swope and W. F. Ames, "Vibrations of a moving threadline," Journal of The Franklin Institute, vol. 275, no. 1, pp. 36-55, 1963.

[7] S. Pracek, "Theory of string motion in the textile process of yarn unwinding," International Journal of Nonlinear Sciences and Numerical Simulation, vol. 8, no. 3, pp. 451-460, 2007.

[8] G. Zhou, X. Sun, and Y. Wang, "Multi-chain digital element analysis in textile mechanics," Composites Science and Technology, vol. 64, no. 2, pp. 239-244, 2004.

[9] Y. Mahadik and S. Hallett, "Finite element modelling of tow geometry in 3D woven fabrics," Composites Part A: Applied Science and Manufacturing, vol. 41, no. 9, pp. 1192-1200, 2010.

[10] S. Gao, B. Liang, and E. Vidal-Salle, "Development of a new 3D beam element with section changes: The first step for large scale textile modelling," Finite Elements in Analysis and Design, vol. 104, pp. 80-88, 2015.

[11] E. Carrera and G. Giunta, "Refined beam theories based on a unified formulation," International Journal of Applied Mechanics, vol. 2, no. 1, pp. 117-143, 2010.

[12] A. Pagani, M. Petrolo, G. Colonna, and E. Carrera, "Dynamic response of aerospace structures by means of refined beam theories," Aerospace Science and Technology, vol. 46, pp. 360373, 2015.

[13] A. Tessler, M. Di Sciuva, and M. Gherlone, "A refined zigzag beam theory for composite and sandwich beams," Journal of Composite Materials, vol. 43, no. 9, pp. 1051-1081, 2009.

[14] Y. Hui, G. Giunta, S. Belouettar, Q. Huang, H. Hu, and E. Carrera, "A free vibration analysis of three-dimensional sandwich beams using hierarchical one-dimensional finite elements," Composites Part B: Engineering, vol. 110, pp. 7-19, 2017.

[15] K. Park, H. Kim, and D. Kim, "Generalized finite element formulation of fiber beam elements for distributed plasticity in multiple regions," Computer-Aided Civil and Infrastructure Engineering, vol. 34, no. 2, pp. 146-163, 2019.

[16] R. Y. Yakoub and A. A. Shabana, “Three dimensional absolute nodal coordinate formulation for beam elements: implementation and applications," Journal of Mechanical Design, vol. 123, no. 4, pp. 614-621, 2001.

[17] W. Yoo, O. Dmitrochenko, S. Park, and O. Lim, "A new thin spatial beam element using the absolute nodal coordinates: application to a rotating strip," Mechanics Based Design of Structures and Machines, vol. 33, no. 3-4, pp. 399-422, 2005.

[18] P. Eberhard and W. Schiehlen, "Computational dynamics of multibody systems: history, formalisms, and applications," Journal of Computational and Nonlinear Dynamics, vol. 1, no. 1, pp. $3-12,2006$

[19] A. A. Shabana, "An absolute nodal coordinates formulation for the large rotation and deformation analysis of flexible bodies," Technical Report, No. MBS96-1-UIC, University of Illinois, Chicago, Ill, USA, 1996.

[20] A. Shabana, "Definition of the slopes and absolute nodal coordinate formulation," Multibody System Dynamics, vol. 1, no. 3, pp. 339-348, 1997.

[21] M. A. Omar and A. A. Shabana, "A two-dimensional shear deformable beam for large rotation and deformation problems," Journal of Sound and Vibration, vol. 243, no. 3, pp. 565-576, 2001.

[22] H. Sugiyama and Y. Suda, "A curved beam element in the analysis of flexible multi-body systems using the absolute nodal coordinates," Proceedings of the Institution of Mechanical Engineers, Part K: Journal of Multi-body Dynamics, vol. 221, no. 2, pp. 219-231, 2007.

[23] O. Dmitrochenko and D. Pogorelov, "Generalization of plate finite elements for absolute nodal coordinate formulation," Multibody System Dynamics, vol. 10, no. 1, pp. 17-43, 2003.

[24] K. Otsuka and K. Makihara, "Absolute nodal coordinate beam element for modeling flexible and deployable aerospace structures," AIAA Journal, vol. 57, no. 3, pp. 1343-1346, 2019.

[25] A. A. Shabana, Computational Continuum Mechanics, Cambridge University Press, Cambridge, UK, 2nd edition, 2012.

[26] Y. Z. Liu and Y. D. Qian, "The experiment of measurement the air drag coefficient of different shape objects," College Physics, vol. 36, no. 3, pp. 16-19, 2017.

[27] Y. Z. Jin and J. Li, "Numerical simulation on coupling of fiber and air flow," Journal of Textile Research, vol. 36, no. 1, pp. 153157, 2015.

[28] S. Y. Chen, Study on Weft Yarns Instability Behavior during The Start-Up of Weft Insertion, Zhejiang Sci-Tech University, Hangzhou, Zhejiang, China, 2015.

[29] Z. G. Pei, Study on the Characteristics and Application of the Fiber-Airflow Interaction in Vortex Spinning, Donghua University, Shanghai, China, 2011. 
[30] H. Lin, Dynamical Behavior and Control of Moving Yarns, Soochow University, Su Zhou, Jiang Su, China, 2014.

[31] A. Abdel-Nasser, A. A. Mohamed, and J. Liu, "The three dimensional gradient deficient beam element (BEAM9) using the absolute nodal coordinate formulation," in Proceedings of the ASME 2014 International Design Engineering Technical Conferences and Computers and Information in Engineering Conference, American Society of Mechanical Engineers, Buffalo, NY, USA, 2014.

[32] Y. Zhang, C. Wei, Y. Zhao, C. Tan, and Y. Liu, "Adaptive ANCF method and its application in planar flexible cables," Acta Mechanica Sinica, vol. 34, no. 1, pp. 199-213, 2018.

[33] L. Wang, Y. Wang, A. M. Recuero, and A. A. Shabana, "ANCF Analysis of Textile Systems," Journal of Computational and Nonlinear Dynamics, vol. 11, no. 3, article 031005, pp. 1-13, 2016.

[34] GB/T 14343-2008, "Testing method for linear density of manmade filament yarns, Chinese National Standards," 2008. 


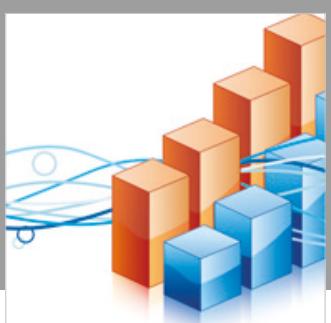

Advances in

Operations Research

\section{-n-m}
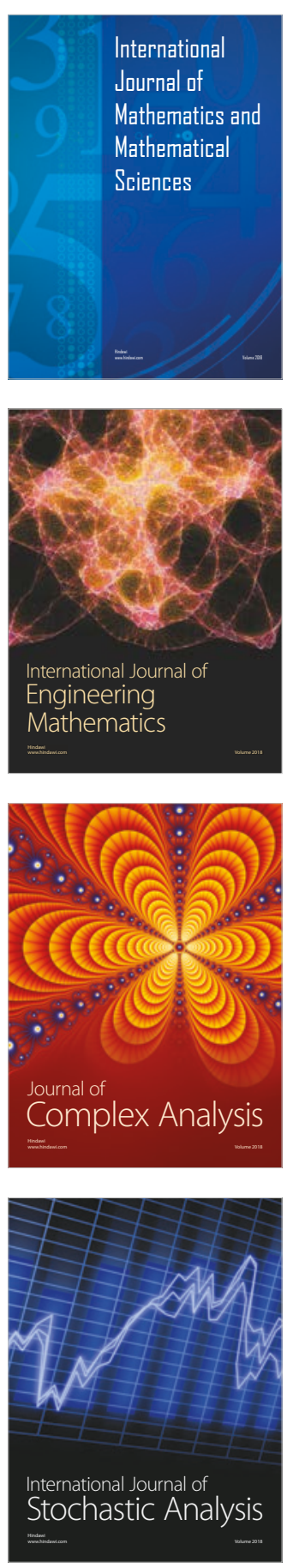
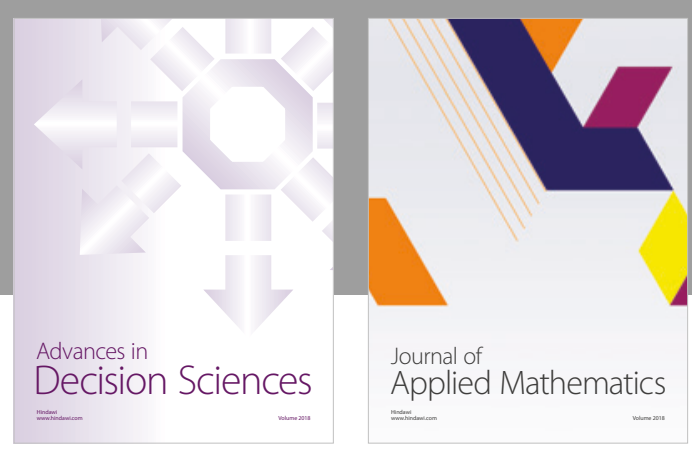

Journal of

Applied Mathematics
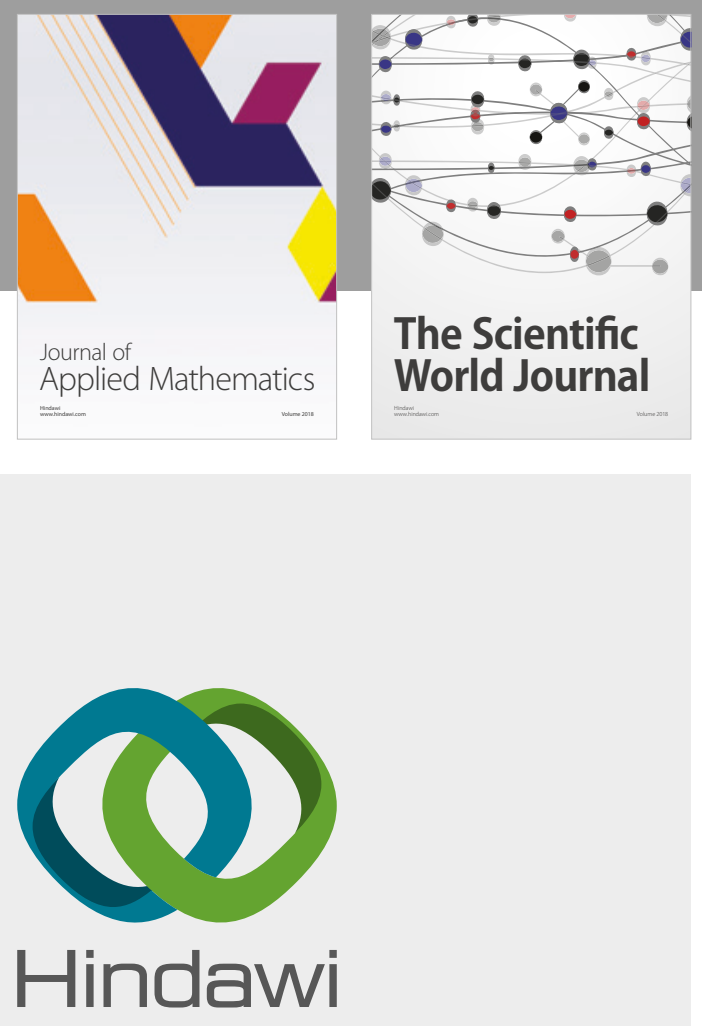

Submit your manuscripts at

www.hindawi.com

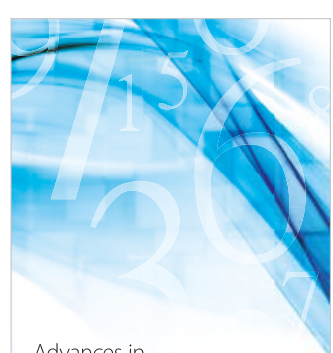

Advances in
Numerical Analysis
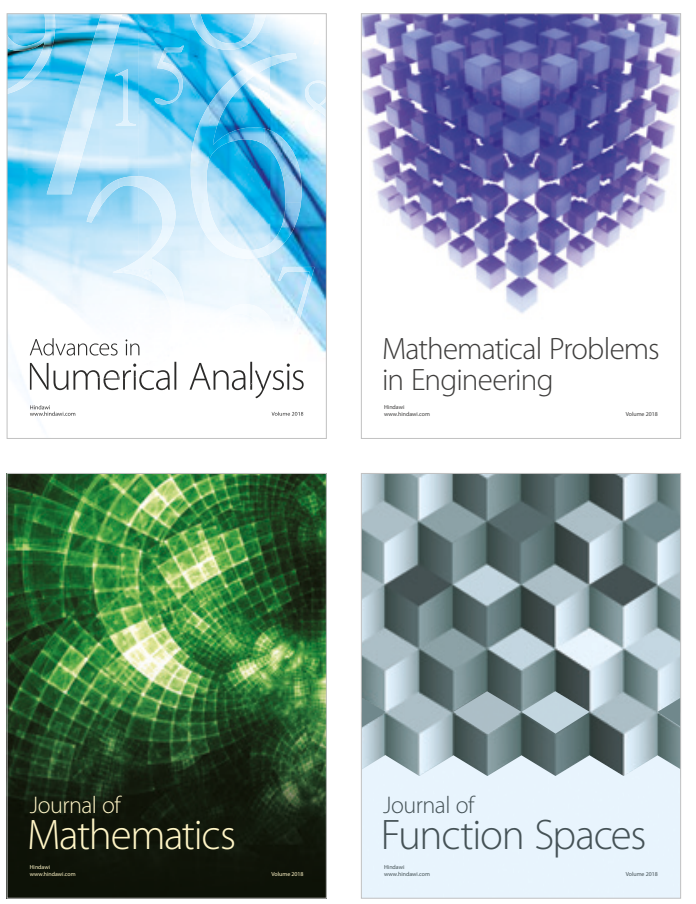

Mathematical Problems in Engineering

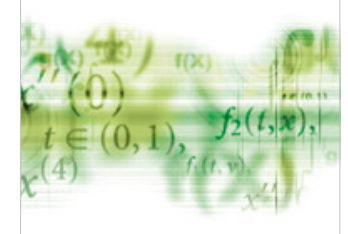

International Journal of

Differential Equations

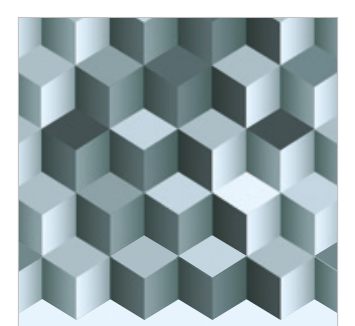

Journal of

Function Spaces

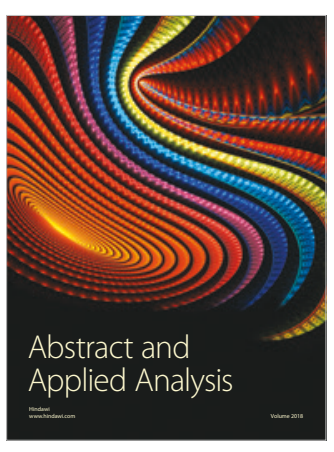

The Scientific

World Journal

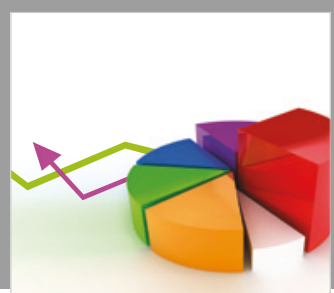

Journal of

Probability and Statistics
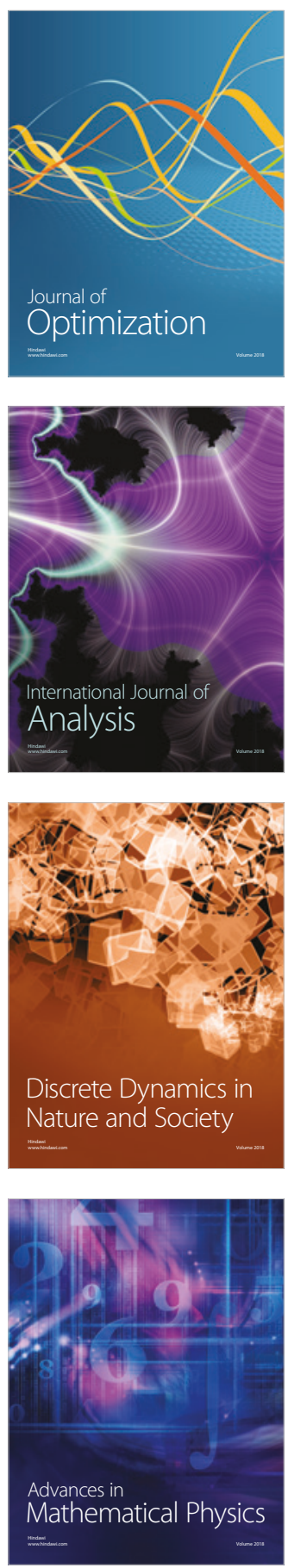